\title{
On Smith Predictor-Based Controller Design for Systems with Integral Action and Time Delay
}

\author{
Ug̃ur Taşdelen \\ Dept. of Electrical \& Electronics Eng. \\ Bilkent Univ. Ankara, 06800 Turkey \\ ugurt@ee.bilkent.edu.tr
}

\author{
Hitay Özbay \\ Dept. of Electrical \& Electronics Eng. \\ Bilkent Univ. Ankara 06800 Turkey \\ hitay@bilkent.edu.tr
}

\begin{abstract}
A new Smith predictor based controller is proposed for systems with integral action and flexible modes under inputoutput time-delay. The design uses controller parametrization and aims to achieve a set of performance and robustness objectives. Compared to existing Smith predictor based designs, disturbance attenuation property is improved, with respect to periodic disturbances at a known frequency. A two-degree of freedom controller structure is shown to be helpful in shaping the transient response under constant reference inputs. Stability robustness properties of this system are also investigated. Simulation results demonstrate the effectiveness of the proposed controller.
\end{abstract}

\section{INTRODUCTION}

Time delay appears frequently in process control systems. Typically, presence of time delay in processes makes it difficult to design a control system. A feedback system with time delay in the loop is a special case of infinite dimensional systems having infinitely many poles. In 1957, O.J.Smith, [25], introduced a special controller structure where the transfer function from reference input to the output can be shaped by designing a controller for the delay free part of the plant. This is a model based structure which uses the advantage of a mathematical model of the process in a minor feedback loop.

Over the last 50 years, many modifications to the Smith predictor structure have been proposed to extend the idea to a wider class of plants. For example, [26] proved that the Smith predictor cannot reject the load disturbance for processes with integration and also showed that there was a steady state error for a load change if the process delay time deviated from its nominal value. Since then, many other modifications have been pointed out to overcome the problem of controlling a process with integrator and dead time, e.g., [19], modified the structure of [26] by adding a filter. Also, [2] proposed a new structure for the control of integrator and dead time processes which decouples the disturbance response from the set-point response. The modifications of [16]-[17], include additional feedback path from the difference of plant output and the model output to the control input. Similarly, [14], proposed a simple relay auto-tuning method for the Smith Predictor and they computed a reduced order process model in terms of a first- or second-order dynamics plus delay time (FOPDT and SOPDT respectively). In the late of 1990's, the limitations of PID controllers controlling resonant, integrating and unstable plants in a conventional feedback structure have been studied
[9], [21]. See [24] for the details of the most of the above mentioned "modified Smith predictor-based controllers" and further references.

In all afore mentioned works, the robustness issue was not explicitly analyzed. In fact, even if the Smith predictor is nominally stable, it is possible to destabilize the feedback system by a minor change in the process dynamics. For example, [11] defined a single multiplicative perturbation to represent the uncertainty in several process parameters. A geometric method is presented in [18] to describe the impacts of the delay uncertainty on the stability of a standard Smith predictor. Also, [8] used system identification method to find out a nominal model and they determined uncertainty bound of the nominal model in the frequency domain through the uncertainty quantification method. A robust criterion for the Smith predictor was also derived in [8]. Mismatch in time delay is analyzed in [1]. Many other researchers also focused on robustness of Smith predictor, see e.g., [10] and [13]. The Smith predictor structure is used in many application areas such as telecommunication [12], [15], [4], biological systems, [23], and flexible-link robot manipulator [3].

In this paper, controller in the structure of Smith predictor will be designed for a flexible robot arm including integrator and time delay, with performance and robustness considerations. Controller parametrization allows widest freedom in choosing controller parameters and this results in improved performance, both in set-point response and disturbance rejection. For the controller obtained in this manner, stability robustness is also investigated. Simulation results show that improved performance can be obtained in the presence of unmodeled dynamics.

The paper is organized as follows. Structure of the plant considered and proposed Smith predictor based controller structure are defined in Section 2. Section 3 analyzes performance with respect to set-point tracking and disturbance rejection. Stability robustness analysis is done in Section 4. Concluding remarks are made in Section 5.

\section{Plant Structure and Controller Design}

A typical flexible robot arm can be represented as in Fig. 1. Control input is the torque applied by the motor and the angular velocity is taken to be the output. Hence, from the physical laws, transfer function of this plant includes an 
integrator. Due to flexibility of the robot arm, high frequency dynamics also enter into the plant transfer function. Time delays in the system appear due to sampling, sensor/actuator non-collocation, and signal transmission depending on the physical distance between the controller and the plant.

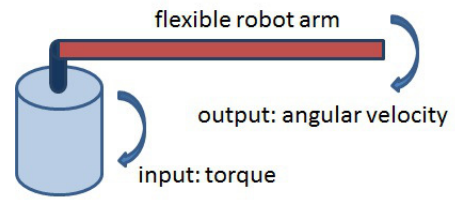

Fig. 1. Representation of a Flexible Robot Arm

There are many approaches to modeling and system identification for flexible robot arm, see e.g. [6],[22] and their references. We will assume that nominal parameters for the flexible modes are obtained from parameter estimation, and any non-minimum phase part is absorbed into the time delay. Hence the plant transfer function from torque to angular velocity is in the form

$$
P_{0}(s)=\frac{K}{s} R_{0}(s) e^{-T_{d} s}
$$

where the gain $K>0$ is proportional to the inertia (mechanical signal amplifiers and scaling factors in the actuator also contribute to the gain), $T_{d}>0$ is the time delay, and $R_{0}(s)$ is a minimum phase transfer function in the form

$$
R_{0}(s)=\frac{\omega_{0}^{2}}{s^{2}+2 \zeta_{0} \omega_{0} s+\omega_{0}^{2}} \prod_{k=1}^{n} \frac{\left(s^{2} / \tilde{\omega}_{k}^{2}\right)+2 \tilde{\zeta}_{k}\left(s / \tilde{\omega}_{k}\right)+1}{\left(s^{2} / \omega_{k}^{2}\right)+2 \zeta_{k}\left(s / \omega_{k}\right)+1}
$$

where $0<\omega_{0}<\tilde{\omega}_{k}<\omega_{k}$ are the resonant and anti-resonant frequencies, and $\tilde{\zeta}, \zeta$, are the damping factors, taking values between 0 and 1 . It is assumed that the above parameters are estimated from system identification, but when it comes to stability robustness analysis uncertainty in $R_{0}(s)$ will be considered. Note that $R_{0}(j \omega) \approx 1$ for all $0 \leq \omega \ll \omega_{0}$.

The structure of proposed Smith predictor based controller for this model is shown in Fig. 2. As seen from Fig. 2, the controller $C_{1}$ is defined as

$$
C_{1}(s)=\frac{\widehat{R}_{\varepsilon}(s)^{-1}}{\widehat{K}}\left(\frac{C_{0}(s)}{1+C_{0}(s) \frac{1-e^{-\widehat{T}_{d}} s}{s}}\right)
$$

Here $\widehat{R}_{\varepsilon}^{-1}(s)=\widehat{R}_{0}^{-1}(s) /(1+\varepsilon s)^{2}$ is the approximate inverse of the term due to flexible modes, with $0<\varepsilon \ll \omega_{n}^{-1}$. $\widehat{R}_{0}^{-1}$ is in the same form of $R_{0}(s)$ except that its parameters are the estimated values of $\omega_{i}, \zeta_{i}, \tilde{\omega}_{i}, \tilde{\zeta}_{i}$ for $i=0,1 \ldots, n$ which are not necessarily matching the exact values used in $R_{0}(s)$. The free part of the controller is $C_{0}(s)$ and it is to be designed from the non-delayed part of the plant as usual in Smith predictor based design. Typically $H(s)=1$ and does not play a role in the feedback system stability analysis, nor in the disturbance attenuation problem. When two-degree of freedom controller scheme is considered, the stable filter $H(s)$ is designed to improve the tracking performance.

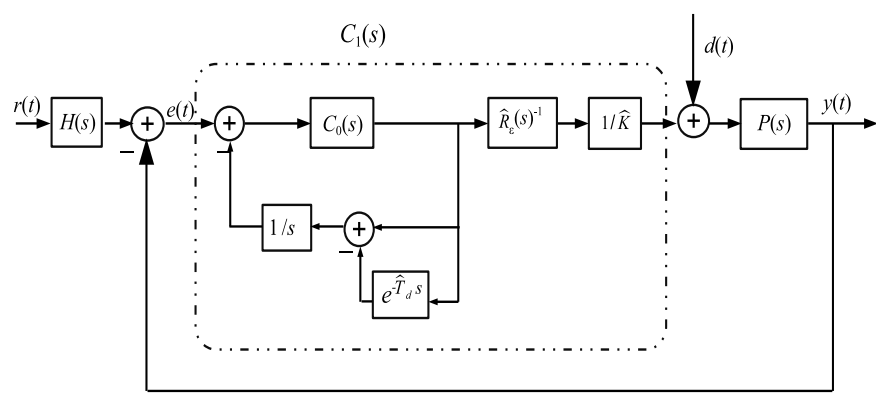

Fig. 2. Proposed Smith Predictor Based Controller Structure

For the plant given in (1), the controller $C_{1}(s)$ is required to satisfy these three conditions:

1) $C_{1}(s)$ must be Type 1 , for perfect steady state tracking of constant reference input $r(t)$.

2) Periodic disturbances $d(t)$ with known frequency, $\omega_{d}$, must be rejected in steady state.

3) The feedback system must be stable with "good" robustness properties (in the sense to be discussed below).

To satisfy first condition, $C_{1}(s)$ is entailed to have a pole at $s=0$, which is important to avoid to steady state error and reject load disturbance. This condition is translated to

$$
\lim _{s \rightarrow 0} C_{1}(s)=\infty \Longrightarrow \lim _{s \rightarrow 0}\left(1+C_{0}(s) \frac{\left(1-e^{-\widehat{T}_{d} s}\right)}{s}\right)=0
$$

From the L'Hôpital Rule, we obtain $1+\widehat{T}_{d} C_{0}(0)=0$ which means

$$
C_{0}(0)=-\frac{1}{\widehat{T}_{d}} .
$$

This is the first design criterion.

According to internal model principle, [5], to satisfy the second condition, $C_{1}(s)$ must have poles at $s= \pm j \omega_{d}$ (since the system is real, only one interpolation condition is sufficient):

$$
\lim _{s \rightarrow j \omega_{d}} C_{1}(s)=\infty \Longrightarrow \lim _{s \rightarrow j \omega_{d}}\left(1+C_{0}(s) \frac{\left(1-e^{-\widehat{T}_{d} s}\right)}{s}\right)=0
$$

which means

$$
C_{0}\left(j \omega_{d}\right)=\frac{-j \omega_{d}}{1-e^{-j \widehat{T}_{d} \omega_{d}}} .
$$

In the same way, this is the second design criterion.

Keeping in mind the above conditions, stability of the feedback system must be guaranteed. With the controller structure $C_{1}(s)$, when the plant is known $P(s)=P_{0}(s)$, the characteristic equation of closed-loop system is

$$
1+C_{0}(s) \frac{1}{s}=0
$$

which means that $C_{0}(s)$ must be designed to stabilize $\frac{1}{s}$, the integrator. If $P_{1}(s)=\frac{1}{s}$, then the set of all controllers which stabilizes $P_{1}$ is found by using controller parametrization, [27]. 
To find this set, let $P_{1}(s)=\frac{N_{p}(s)}{D_{p}(s)}$, where $D_{p}(s)=\frac{s}{s+a}$ and $N_{p}(s)=\frac{1}{s+a}$ with $a>0$ is a parameter to be chosen via pole placement method as shown below.

All stabilizing controllers for $P_{1}(s)$ are parameterized as:

$$
C_{0}(s)=\frac{X(s)+D_{p}(s) Q(s)}{Y(s)-N_{p}(s) Q(s)}
$$

where $Q \in H_{\infty}$ and $Q \neq Y N_{p}^{-1}$. Here, $X, Y \in H_{\infty}$ are functions satisfying

$$
N_{p}(s) X(s)+D_{p}(s) Y(s)=1
$$

It is clear from (7) that

$$
Y(s)=\frac{1-N_{p}(s) X(s)}{D_{p}(s)}
$$

Since $D_{p}(0)=0, X(0)$ must be equal to $\frac{1}{N_{p}(0)}$ which means $X(0)=a$. Since $X(s)$ should be stable, simply it can be chosen as $X(s)=a$. Then, from (8), $Y$ can be found as:

$$
Y(s)=\frac{1-\frac{1}{s+a} a}{\frac{s}{s+a}}=\frac{(s+a)-a}{s}=1
$$

If all functions are put into (6),

$$
C_{0}(s)=\frac{a+\frac{s}{s+a} Q(s)}{1-\left(\frac{1}{s+a}\right) Q(s)} .
$$

Now, the problem is reduced to finding a stable $Q(s)$ satisfying the interpolation conditions (3)-(4). From (9) the interpolation conditions are translated to

$$
\begin{gathered}
Q(0)=a\left(1+a \widehat{T}_{d}\right) \\
Q\left(j \omega_{d}\right)=\frac{\left.\left(j \omega_{d}+a-a e^{-j \omega_{d} \widehat{T}_{d}}\right)\right)\left(j \omega_{d}+a\right)}{\left(j \omega_{d}\right) e^{-j \omega_{d} \widehat{T}_{d}}} .
\end{gathered}
$$

To satisfy (10) and (11), a second order transfer function in the form

$$
Q(s)=\frac{b s^{2}+c s+d}{s^{2}+e s+f}
$$

will be postulated for $Q(s)$. Here $e, f>0$ are free parameters; once these free parameters are chosen, $b, c$ and $d$ are determined from the interpolation conditions (10) and (11). As a result, $C_{0}(s)$ turns into

$$
C_{0}(s)=\frac{(a+b) s^{3}+\left(l_{1} a+c\right) s^{2}+\left(l_{2} a+d\right) s+a l_{3}}{s^{3}+\left(l_{1}-b\right) s^{2}+\left(l_{2}-c\right) s+\left(l_{3}-d\right)}
$$

where $l_{1}=e+a, l_{2}=f+a e, l_{3}=a f$.

With the above design, when $P=P_{0}, \widehat{K}=K, \widehat{T}_{d}=T_{d}$, $\widehat{R}_{0}=R_{0}$ and $\varepsilon \rightarrow 0$, the closed-loop transfer function from $r$ to $y$ in Fig. 2, is $T_{r y}(s)=T_{0}(s) H(s)$ where $T_{0}=P_{0} C_{1}(1+$ $\left.P_{0} C_{1}\right)^{-1}$, and it reduces to

$$
\begin{aligned}
T_{0}(s) & =\frac{N_{T}(s)}{\left(s^{2}+e s+f\right)(s+a)^{2}} e^{-T_{d} s} \\
N_{T}(s) & =a(s+a)\left(s^{2}+e s+f\right)+s\left(b s^{2}+c s+d\right)
\end{aligned}
$$

where $a>0, e>0$ and $f>0$ are chosen to place the closed loop system poles at the desired locations, and $b, c, d \in \mathbb{R}$ are determined from the interpolation conditions (10) and (11).
The pre-filter $H(s)$ can now be designed to cancel some of the higher dynamics in $T_{0}(s)$ depending on the location of the zeros of $N_{T}(s)$. Typically, we choose a stable and strictly proper $H(s)$ with $H(0)=1$. One particular choice is

$$
H(s)=\frac{1}{1+\tau s}
$$

where $\tau>0$ is the free design parameter, typically it is designed to cancel the fastest negative real axis zero of $T_{0}(s)$. This idea can be extended to define a possibly higher order $H(s)$ to shape $\left|T_{0}(j \omega)\right|$.

\section{Performance Analysis}

This section will be divided into two parts. Performance will be analyzed in terms of set-point response and then disturbance rejection. Proposed controller is compared with the alternative Smith predictor based controller of Mataušek and Micić, [17], which is proved to offer good performance (many recent application oriented papers in this area consider [17] as the baseline for comparison, see e.g. [7] and [24]). In this section, it is assumed that $P=P_{0}, \widehat{K}=K, \widehat{T}_{d}=T_{d}$, $\widehat{R}_{0}=R_{0}$ and $\varepsilon \rightarrow 0$ for nominal system performance analysis. The effects of mismatch in these parameters will be discussed in Section IV. The plant taken into consideration is

$$
P_{0}(s)=\frac{20}{s} R_{0}(s) e^{-0.2 s}
$$

where $R_{0}$ includes flexible modes

$$
R_{0}(s)=\frac{\left(\frac{s}{\tilde{\omega}_{1}}\right)^{2}+2 \frac{\tilde{\zeta}_{1}}{\tilde{\omega}_{1}} s+1}{\left(\left(\frac{s}{\omega_{1}}\right)^{2}+2 \frac{\zeta_{1}}{\omega_{1}} s+1\right)\left(\left(\frac{s}{\omega_{0}}\right)^{2}+2 \frac{\zeta_{0}}{\omega_{0}} s+1\right)}
$$

with the values $\tilde{\omega}_{1}=115, \tilde{\zeta}_{1}=0.22, \omega_{1}=125, \zeta_{1}=0.06$, $\omega_{0}=95, \zeta_{0}=0.15$.

For this plant the designed parameters are $a=1, e=2$, $f=2$ that leads to $b=3.88, c=0.986, d=2.4$, and

$$
C_{0}(s)=\frac{4.88(s+0.355)\left(s^{2}+0.44 s+1.16\right)}{(s-0.14)\left(s^{2}-0.74 s+2.90\right)} .
$$

Thus we have $T_{r y}(s)=T_{0}(s) H(s)$ where

$$
T_{0}(s)=\frac{4.88(s+0.355)\left(s^{2}+0.44 s+1.16\right)}{\left(s^{2}+2 s+2\right)(s+1)^{2}} e^{-0.2 s} .
$$

We select $H(s)=(1+s / 0.355)^{-1}$ in the setpoint analysis given below. By a proper choice of $H$, it is also possible to cancel the lightly damped zeros of $T_{0}(s)$; but this should be avoided, because location of these zeros are very sensitive to plant parameters used in the interpolation conditions (10)(11). Note that $H(s)$ does not play a role in the disturbance attenuation analysis; and it will be taken as $H(s)=1$ for robust stability analysis.

\section{A. Setpoint Response Analysis}

Responses of the proposed controller and the alternative Smith Predictor-based controller design of [17] are given in Fig. 3. The proposed controller results in a faster response: $2 \%$ settling time of $7.1 \mathrm{sec}$. versus $13.1 \mathrm{sec}$. Since proposed controller has three free parameters, it is possible to further optimize the setpoint response. 


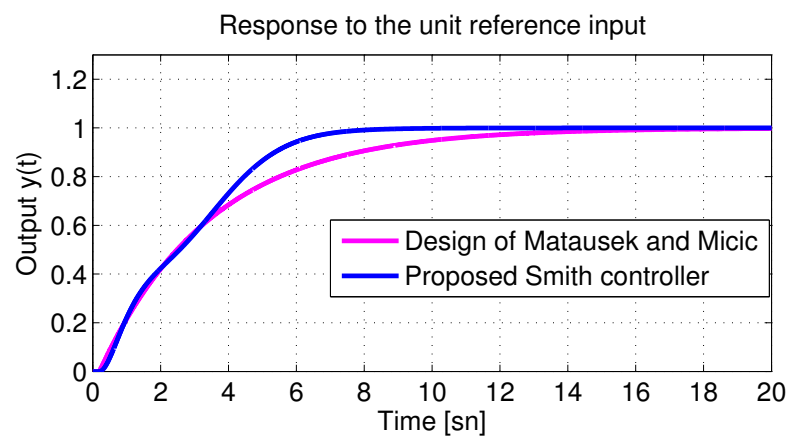

Fig. 3. Setpoint Responses

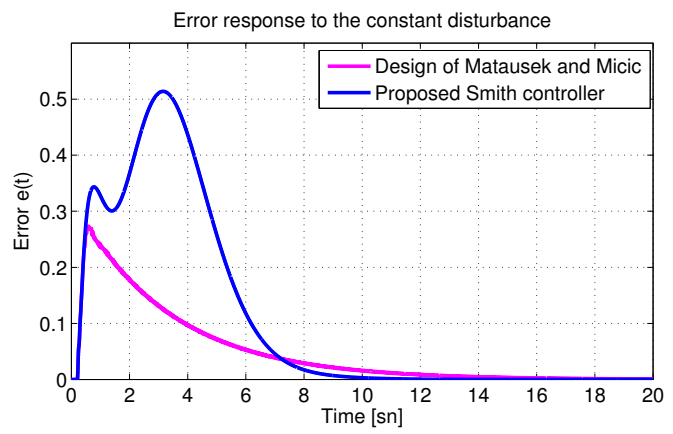

Fig. 4. Response to the constant disturbances under no reference input

\section{B. Disturbance Rejection Analysis}

The simulations are performed for two different disturbance types: (i) constant signal and (ii) a periodic signal of frequency $\omega_{d}=1.5 \mathrm{rd} / \mathrm{sec}$. By using two degree of freedom controller structure, Mataušek and Micić, [17], provide fast disturbance rejection for constant load disturbances which is caused by derivative action and fast estimation of the disturbance signal. However, [17] fails to reject sinusoidal disturbances. Since proposed controller has poles at $s=0$ and $s= \pm j \omega_{d}$, where $\omega_{d}$ is the frequency of sinusoidal disturbance, Fig. 4 and Fig. 5 show that constant and sinusoidal disturbances are suppressed in steady state as expected.

\section{STABILITY/ROBUSTNESS ANALYSIS}

In order to determine the stability margins, open loop transfer function $G(j \omega)$ is analyzed:

$$
\begin{aligned}
G(s) & =P_{0}(s) C_{1}(s) \\
& =\frac{K}{s} R_{0}(s) e^{-T_{d} s}\left(\frac{C_{0}(s)}{1+C_{0}(s) \frac{\left(1-e^{-\widehat{T}_{d} s}\right)}{s}}\right) \frac{1}{\widehat{K}} \widehat{R}_{\varepsilon}^{-1}(s) \\
& =\left(\frac{K}{\widehat{K}}\right)\left(R_{0}(s) \widehat{R}_{\varepsilon}^{-1}(s)\right) \frac{C_{0}(s) e^{-T_{d} s}}{s+C_{0}(s)\left(1-e^{-\widehat{T}_{d} s}\right)}
\end{aligned}
$$

Let $\widehat{T}_{d}=T_{d}$ and define

$$
G_{0}(s)=\frac{C_{0}(s) e^{-\widehat{T}_{d} s}}{s+C_{o}(s)\left(1-e^{-\widehat{T}_{d} s}\right)} .
$$

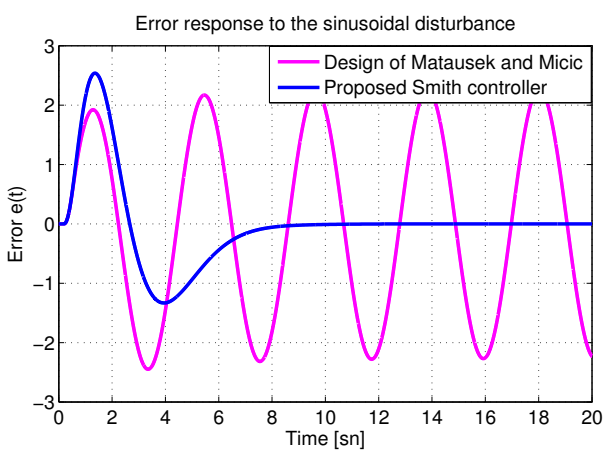

Fig. 5. Response to the sinusoidal disturbances under no reference input

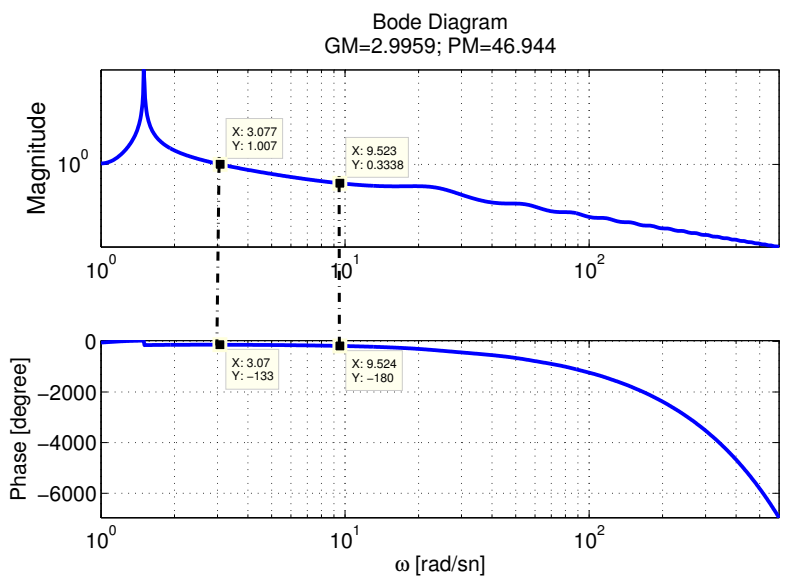

Fig. 6. Frequency response of $G_{0}$

The case $R_{0}(s) \widehat{R}_{\varepsilon}^{-1}(s) \neq 1$ will be considered later at the end of this section.

The gain and phase margins obtained from Bode Plot of $G_{0}(j \omega)$ give the information on how much uncertainty in the gain $(K / \widehat{K})$ and delay mismatch $\left(T_{d}-\widehat{T}_{d}\right)$ can be tolerated, [20]. Fig. 6 shows the stability margins on the Bode plots, $G M=3 \equiv 9.5 d B, P M=47^{\circ}$ and $D M=0.27$ sec. It should be noted that, stability margins can be improved by changing free parameters. However, this may deteriorate the setpoint tracking and disturbance rejection performances.

The best way to analyze the robustness in the presence of both gain and phase perturbation is the vector margin (VM), which is defined as the distance between the critical point, -1 , and $G_{0}(j \omega)$ :

$$
V M=\min _{\omega}\left|1+G_{0}(j \omega)\right|
$$

For the system designed, $V M=0.625$, which is relatively large for good stability robustness. Robustness to variations in the gain $K$ and delay $T_{d}$ is analyzed by calculating the VM when these parameters are fixed as $\widehat{K}=20, \widehat{T}_{d}=0.2$ sec in the controller but they are modified in the plant, taking values in the intervals $K \in[1,60]$ and $T_{d} \in[0,0.55] \mathrm{sec}$, see Fig. 7. This figure also shows the stability boundary (where $V M=0)$ and the nominal operating point. 


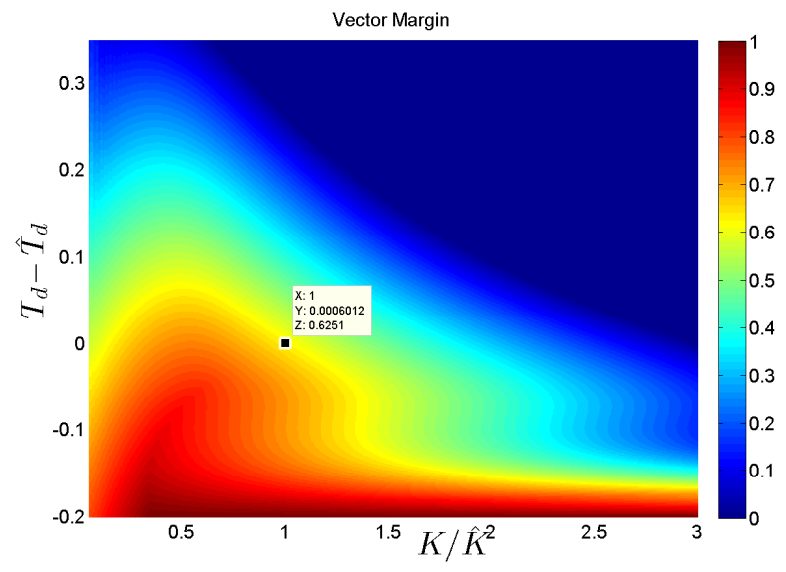

Fig. 7. Vector Margin for different $K$ and $T_{d}$

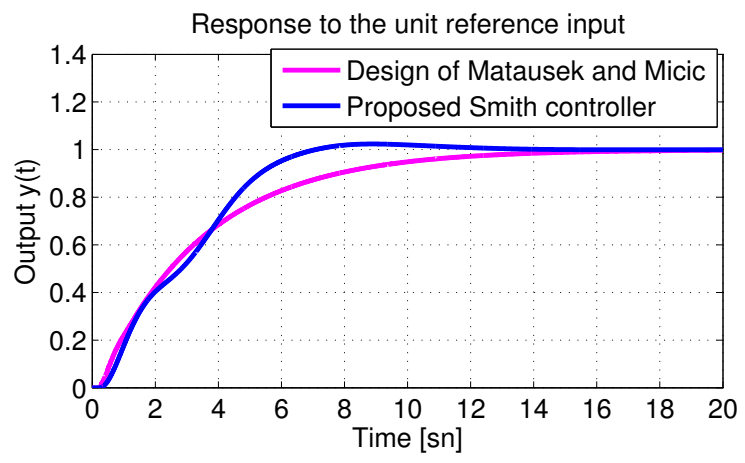

Fig. 8. Setpoint Response for $\widehat{K}=28$ and $\widehat{T}_{d}=T_{d}$

In controller design, using Fig. 7 effectively can be very useful to increase robustness. For example, Fig. 7 shows that if the ratio $(K / \widehat{K})$ is decreased to 0.7 (i.e. $\widehat{K}$ is chosen as $28.5)$ vector margin becomes 0.715 , assuming that $\widehat{T}_{d}=T_{d}$. That leads to $G M=4.26, P M=47^{\circ}, D M=0.33 \mathrm{sec}$. With this modification better stability margins are obtained with the expense of slight performance loss: the corresponding setpoint response and disturbance responses are given in Fig. 8 and Fig. 9 respectively.

In order to analyze stability/robustness in the presence of dynamic uncertainty, consider the plant

$$
P(s)=P_{0}(s)\left(1+\Delta_{m}(s)\right)
$$

where $\Delta_{m}(s)$ is multiplicative uncertainty, which is assumed to be stable. The feedback system formed by the nominal controller designed as above and the uncertain plant (22) is robustly stable if and only if

$$
\left|\Delta_{m}(j \omega)\right|<\frac{1}{\left|T_{0}(j \omega)\right|} \quad \forall \omega,
$$

where $T_{0}(s)$ is as in (19). Recall that there are 8 parameters in the plant (17)-(18); varying each one of these will give a plant in the form (22), with a corresponding $\Delta_{m}(j \omega)$. Considering $20 \%$ variation in the nominal values of these 8 parameters
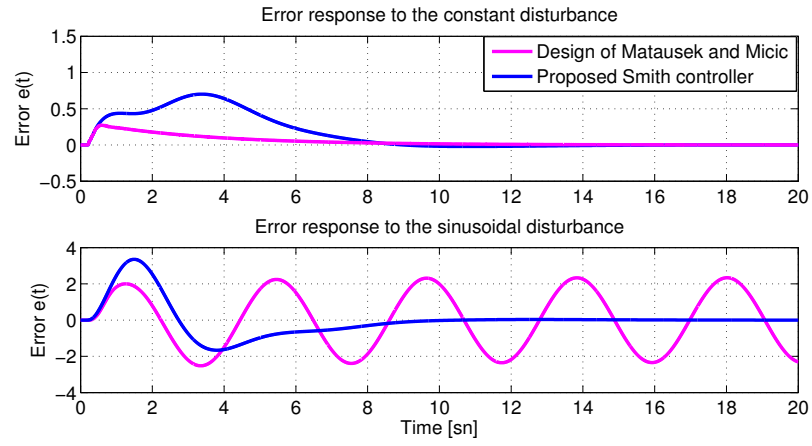

Fig. 9. Disturbance Responses for $\widehat{K}=28$ and $\widehat{T}_{d}=T_{d}$

we obtain a family of $\Delta_{m}$. Fig. 10 shows that all of these $\left|\Delta_{m}(j \omega)\right|$ (red lines) remain below the graph of $1 /\left|T_{0}(j \omega)\right|$, hence satisfying the robust stability inequality (23). Moreover, the gap between the red and blue lines represent how much additional uncertainty can be tolerated at each frequency.

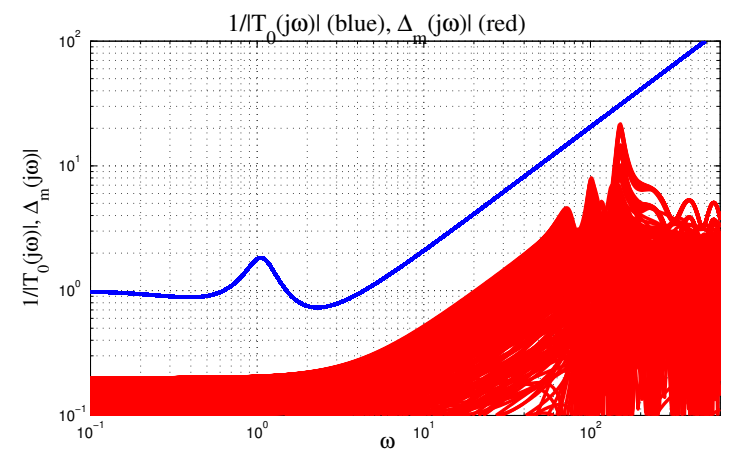

Fig. 10. Illustration of robust stability.

Let us now ignore the flexible modes in the controller design, $\widehat{R}_{\varepsilon}(s)=1$, but include $R_{0}(s)$ in the plant. Also, consider $25 \%$ uncertainty in the gain and time delay, i.e., $K=1.25 \widehat{K}, T_{d}=1.25 \widehat{T}_{d}$. The setpoint response for this case is as shown in Fig. 11. It is observed that the proposed controller is robust to these perturbations. On the other hand, the benchmark controller of [17] shows an unstable response for combined perturbations in the delay and gain, with the presence of the flexible modes $R_{o}(s)$ in the plant. The reason for this behavior can be explained by Fig. 12, where robustness inequality (23) is satisfied with the present controller, but it is violated with the controller of [17].

\section{CONCLUSIONS}

A Smith predictor based controller structure is considered. Based on interpolation conditions imposed by constant reference tracking, and periodic disturbance rejection, the free part of the controller, $C_{0}(s)$ is designed. The resulting $C_{0}(s)$ is a third order transfer function. In this design there are three free parameters $a, e$, and $f$; they determine the closed loop 


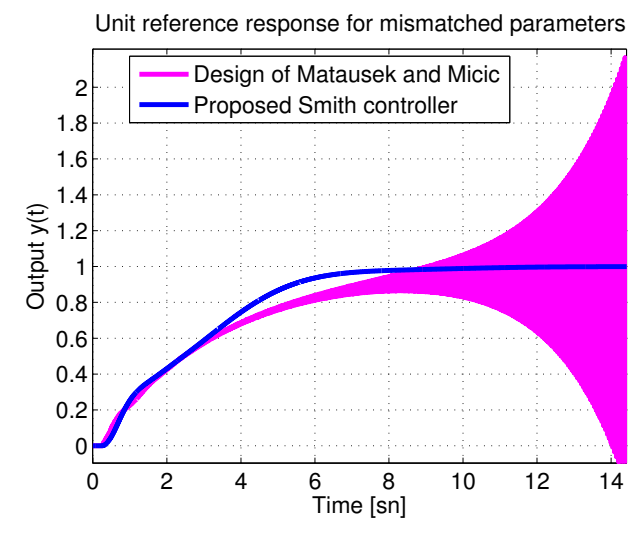

Fig. 11. Setpoint Response for Mismatched Parameters.

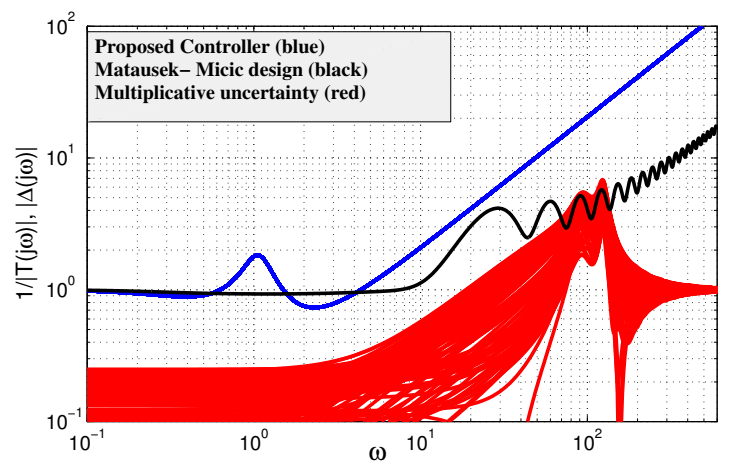

Fig. 12. Robust stability check under $25 \%$ uncertainty in $T_{d}$ and $K$; here $R_{0}(s)$ have fixed parameters but it is treated as unmodeled dynamics.

system pole locations. Optimization of these parameters for other performance and robustness measures is possible.

In the implementation of the overall controller $C_{1}(s)$, shown in Fig. 2, the feedback loop around $C_{0}$ is a filter whose impulse response is finite duration $\frac{1-e^{-\widehat{T}_{d} s}}{s}$. So, this component can be implemented easily in a numerically reliable manner. The controller also uses the (approximate) inverse of the stable minimum phase part of the plant, $1 /\left(K R_{\varepsilon}(s)\right)$. Robustness to uncertainties in $K, T_{d}$ and the parameters of $R_{0}(s)$ is also demonstrated. If an upper bound of multiplicative uncertainty is given, then it is possible to use $H_{\infty}$ control techniques to modify the design of $C_{0}$ accordingly.

\section{ACKNOWLEDGMENTS}

This work was supported in part by ASELSAN Inc; the authors would like to thank A. B. Özgüler, Ö. Morgül, S. Güler, E. O. Arı, O. C. Erdog̃an, M. B. Gürcan and B. Bilgin for fruitful discussions within the framework of this project. We also like to acknowledge the partial equipment support provided by the DPT-HAMIT project.

\section{REFERENCES}

[1] Adam, E. J., Crisalle, O. D., Latchman, H. A., "Robustness of the Smith Predictor with Respect to Uncertainty in the Time-Delay Parameter", Proc. American Control Conference, Chicago IL, 2000, pp. 1452-1457.
[2] Astrom, K.J., Hang, C.C., Lim, B.C., "A New Smith Predictor for Controlling a Process with an Integrator and Long Dead-Time", IEEE Transactions on Automatic Control, vol. 39 (1994), pp. 343-346.

[3] Chen, G., Desages, A., Julian, P., "Trajectory tracking and robust stability for a class of time delayed flexible joint robotic manipulators," International Journal of Control, vol. 68 (1997), pp. 259-276.

[4] De Cicco, L., Mascolo, S., Niculescu, S-I., "Robust stability analysis of Smith predictor-based congestion control algorithms for computer networks," Automatica, vol. 47 (2011), pp. 1685-1692.

[5] Francis, B. A., Wonham, W. M., "The internal model principle of control theory," Automatica, Vol. 12, (1976), pp. 457-465.

[6] Fu, K. S., Gonzalez, R. C., Lee, C. S. G., Robotics: Control, Sensing, Vision, and Intelligence, McGraw-Hill, New York, 1987.

[7] Guzmán, J. L., García, P., Hägglund, T., Dormido, S., Albertos, P., Berenguel, M., "Interactive tool for analysis of time-delay systems with dead-time compensators," Control Engineering Practice, vol. 16 (2008), pp. 824-835.

[8] Hong, K. S., Kang, D. H., Kim, J. G., "Robust Smith Predictor Design via Uncertainty Quantification: Application to a Reclaimer," Proc. SYSID 2000: 12th IFAC Symposium on System Identification, Santa Barbara, CA, June 21-23, 2000, pp.575-580,

[9] Kwak, H.J., Sung S.W., Lee I. , "On-line process identification and autotuning for integrating process", Ind. Eng. Chem. Res., vol. 36 (1997), pp. $5329-5338$.

[10] Kristiansson, B., Lennartson, B., "Robust PI and PID Controllers including Smith Predictor Structure," Proceedings of the American Control Conference, Arlington VA, June 2001, pp. 25-27.

[11] Laughlin, D.L., Rivera, D.E., Morari, M., "Smith predictor design for robust performance", Int. J. Control, vol. 46 (1987), pp. 477-504.

[12] Lee, B-K., Chen, H-W., Chen, B-S., "Power Control of Cellular Radio Systems via Robust Smith Prediction Filter," IEEE Transactions on Wireless Communications, vol. 3 (2004), pp. 1822-1831.

[13] Lee, D., Lee, M., Sung, S., Lee, I. , "Robust PID tuning for Smith predictor in the presence of model uncertainty," Journal of Process Control, vol. 9 (1999), pp. 79-85.

[14] Mahji, S., Atherton, D., "Obtaining controller parameters for a new Smith predictor using autotuning," Automatica, vol. 36 (2000), pp. 16511658.

[15] Mascolo, S., "Congestion control in high-speed communication networks using the Smith principle," Automatica, vol. 35 (1999), pp. 19211935.

[16] Mataušek, M. R., Micić, A. D., "A modified Smith predictor for controlling a process with an integrator and long dead-time", IEEE Trans. Autom. Control, vol. 41 (1996), pp. 1199-1203.

[17] Mataušek, M. R., Micić, A. D., "On the modified Smith predictor for controlling a process with an interator and long dead-time," IEEE Trans. Autom. Control, vol. 44 (1999), 1603-1606.

[18] Morarescu, C.-I., Niculescu, S.-I., Gu, K., "On the geometry of stability regions of Smith predictors subject to delay uncertainty," IMA Journal on Mathematical Control and Information, vol. 24 (2007), pp. 411-423.

[19] Normey-Rico, J.E., Camacho, E.F., "Robust tuning of dead-time compensators for processes with an integrator and long dead-time," IEEE Trans. Autom. Control, vol. 44 (1999), pp. 1597-1603.

[20] Özbay, H., Introduction to Feedback Control Theory, CRC Press, Boca Raton FL, 2000

[21] Park J.H., Sung S.W., Lee, I., "An enhanced PID control strategy for unstable processes", Automatica, vol. 34 (1998), pp. 751-756.

[22] Spong, M. W. , Hutchinson, S., Vidyasagar, M., Robot modeling and control. John Wiley \& Sons, Inc., New York, 2006.

[23] Van der Smagt, P., Hirzinger G., "The cerebellum as computed torque model", Proc. of the 4th Int. Conf. on Knowledge-Based Intelligent Engineering Systems and Allied Technologies, Brighton UK, AugustSeptember 2000, pp. 760-763.

[24] Visioli, A., Zhong, Q-C., "Smith-predictor-based Control," in Control of Integral Processes with Dead Time, Advances in Industrial Control, Springer, London 2011, pp. 141-185.

[25] Smith, O. J. M., "Closer control of loops with dead time," Chemical Engineering Progress, vol. 53 (1957), pp. 217-219.

[26] Watanabe, K., Ito, M., "A Process Model Control for Linear Systems with Delay," IEEE Transactions on Automatic Control, vol. 26 (1981), pp. 1261-1265.

[27] Youla, D.C., Jabr, H., Bongiorno, J.J., "Modern Wiener-Hopf design of optimal controllers, Part I," IEEE Trans. Autom. Control, vol. 21 (1976), pp. 3-13. 